\title{
Application of tomographic inversion in studying airglow in the mesopause region
}

\author{
T. Nygrén ${ }^{1}$, M. J. Taylor ${ }^{2}$, M. S. Lehtinen ${ }^{3}$, M. Markkanen ${ }^{3}$ \\ ${ }^{1}$ Department of Physical Sciences, University of Oulu, FIN-90570 Oulu, Finland \\ ${ }^{2}$ Space Dynamics Laboratory and Physics Department, Utah State University, Logan, UT, 84322, USA \\ ${ }^{3}$ Sodankylä Geophysical Observatory, FIN-99600 Sodankylä, Finland
}

Received: 6 October 1997 / Revised: 23 March 1998 / Accepted: 3 April 1998

\begin{abstract}
It is pointed out that observations of periodic nightglow structures give excellent information on atmospheric gravity waves in the mesosphere and lower thermosphere. The periods, the horizontal wavelengths and the phase speeds of the waves can be determined from airglow images and, using several cameras, the approximate altitude of the luminous layer can also be determined by triangulation. In this paper the possibility of applying tomographic methods for reconstructing the airglow structures is investigated using numerical simulations. A ground-based chain of cameras is assumed, two-dimensional airglow models in the vertical plane above the chain are constructed, and simulated data are calculated by integrating the models along a great number of rays with different elevation angles for each camera. After addition of random noise, these data are then inverted to obtain reconstructions of the models. A tomographic analysis package originally designed for satellite radiotomography is used in the inversion. The package is based on a formulation of stochastic inversion which allows the input of a priori information to the solver in terms of regularization variances. The reconstruction is carried out in two stages. In the first inversion, constant regularization variances are used within a wide altitude range. The results are used in determining the approximate altitude range of the airglow structures. Then, in the second inversion, constant non-zero regularization variances are used inside this region and zero variances outside it. With this method reliable reconstructions of the models are obtained. The number of cameras as well as their separations are varied in order to find out the limitations of the method.
\end{abstract}

Key words. Tomography $\cdot$ Airglow $\cdot$ Mesopause $\cdot$ Gravity waves

\section{Introduction}

Gravity waves, particularly small-scale, short-period waves, are known to be important drivers of the mean winds and thermal structure of the mesosphere and lower thermosphere (MLT) region via wave energy and momentum transport (e.g. Fritts and Vincent, 1987). Images of the naturally occurring nightglow emissions provide an excellent technique for quantifying the effects of these waves as they propagate through the MLT region $(\approx 80-100 \mathrm{~km})$, yielding information on their occurrence frequency, horizontal directions of motion and their horizontal scale sizes and phase speeds (e.g. Moreels and Herse, 1977; Taylor et al., 1987, 1995a; Swenson and Mende, 1994). To date, most imaging studies have been made using the bright near-infrared $\mathrm{OH}$ band emission which originates from a well-defined layer centred at $87 \mathrm{~km}$ and exhibiting a night-time halfwidth of $8 \mathrm{~km}$ (e.g. Baker and Stair, 1988). However, with the development of sensitive solid-state CCD imaging systems, measurements of gravity waves in other fainter near-infrared and visible wavelength nightglow emissions such as the OI $(557.7 \mathrm{~nm})$ emission (peak altitude $96 \mathrm{~km}$ ) and the $\mathrm{Na}(589.2 \mathrm{~nm})$ emission (peak altitude $90 \mathrm{~km}$ ) are now practical. As these emissions, particularly the OI layer, reside at heights above the $\mathrm{OH}$ layer they enhance significantly the potential of image measurements for investigating the properties of smallscale gravity waves throughout the MLT region.

In particular, the airglow data offer a unique threedimensional measurement capability for quantifying the effects of small-scale waves on the MLT (e.g. Taylor et al., 1987). In order to optimize these measurements it is therefore essential to have accurate measurements of the height, and height variability of the individual wave structures in each emission layer. Triangulation measurements of wave structure in the $\mathrm{OH}$ nightglow emission (e.g. Peterson and Kieffaber, 1973) and the OI(557.7 nm) emission (e.g. Taylor et al., 1995b) have

Correspondence to: T. Nygrén 
been made to estimate the mean height of the structures but only to an accuracy of typically $\pm 2 \mathrm{~km}$ and with only limited capability for detailed studies of the individual layer morphology. In this paper we investigate the potential of tomographic techniques to study wave structure imaged simultaneously in several nightglow emissions. Tomographic inversion has been used in atmospheric sciences already for a long time both for determining the ionospheric electron density from difference Doppler measurements and the auroral luminosity from auroral camera or photometer observations.

Satellite radio tomography for measuring the ionospheric electron density was suggested by Austen et al., (1986), and it has later been developed in a great number of papers (e.g. Austen et al., 1988; Raymund et al., 1990; Andreeva et al., 1992; Fremouw et al., 1992; Kunitsyn and Tereshchenko, 1992; Pryse and Kersley, 1992; Pryse et al., 1993; Markkanen et al., 1995; Nygrén et al., 1996b). The method has now reached a level which allows its use in ionospheric research (Mitchell et al., 1997).

The first studies of auroral tomography were made using satellite-borne scanning photometers (Solomon et al., 1984, 1985). Later, auroral tomographic studies have been made using ground-based cameras or scanning photometers (Aso et al., 1990; Vallance-Jones et al., 1991; Aso and Ejiri, 1992; Nygrén et al., 1996a, 1997a) and photometers carried by rockets (McDade and Llewellyn, 1991; McDade et al., 1991).

In the field of airglow, tomographic research has been made using the airglow experiment on board the Atmospheric Explorer satellites (Solomon et al., 1988; Abreu and Yee, 1989). These studies, however, are aimed at investigating the vertical airglow profile up in the thermosphere, not the airglow structures lower down around the mesopause altitude. It seems likely that tomographic methods could also be applied in the mesopause region using a set of cameras in a chain on the ground level. The purpose of the present paper is to investigate this possibility using data simulation. It is believed that the optimal number of cameras in the chain as well as their separations can be determined in this way. Thus the simulations would work as a basic study needed in planning an eventual camera network for experimental airglow tomography.

\section{Example observations}

Most nightglow image measurements indicate a predominance for waves with horizontal wavelengths ranging approximately from 5 to $70 \mathrm{~km}$ and exhibiting observed horizontal phase speeds of up to $100 \mathrm{~m} / \mathrm{s}$, yielding observed periods of $\approx 5-60 \mathrm{~min}$ (e.g. Clairemidi et al., 1985; Taylor et al., 1998). The patterns are frequently extensive $\left(>5 \times 10^{6} \mathrm{~km}^{2}\right)$ and have often been attributed to freely propagating gravity waves of vertical wavelengths longer than $10 \mathrm{~km}$ (e.g. Taylor et al., 1987).

Figure 1 shows two examples of short period gravity waves imaged in (a) the near-infrared $\mathrm{OH}$ emission, and (b) the OI $(557.7 \mathrm{~nm})$ emission. The data were obtained from Bear Lake Observatory, Utah $\left(41.6^{\circ} \mathrm{N}, 111.6^{\circ} \mathrm{W}\right)$ using a sensitive, multi-wavelength CCD imager capable of providing quantitative spatial and intensity measurements of gravity wave signatures in the visible and nearinfrared nightglow emissions over a large $\left(180^{\circ}\right)$ field of view. Sequential images of selected nightglow emissions
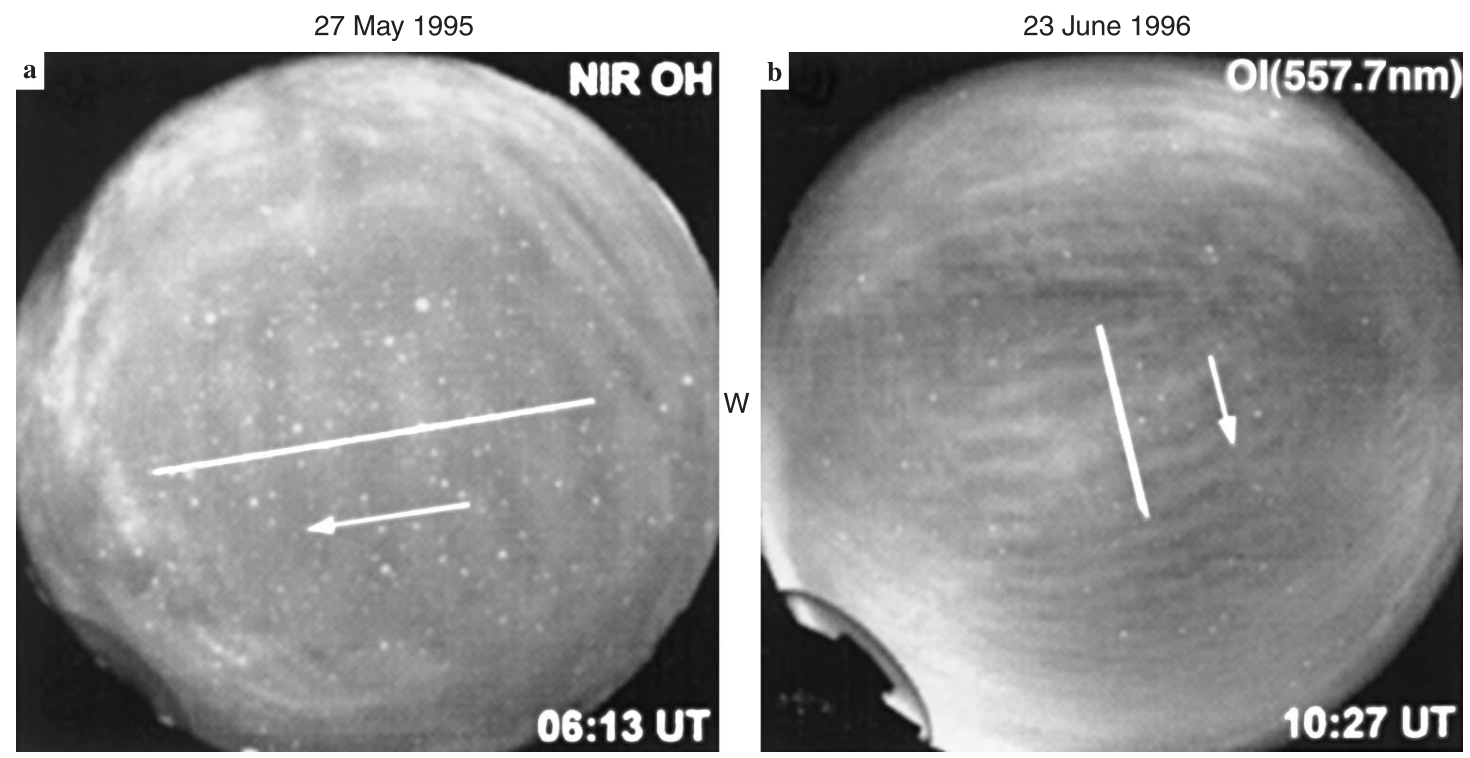

$\mathrm{N}$

Fig. 1a, b. Examples of nightglow images from Bear Lake Observatory, Utah $\left(41.6^{\circ} \mathrm{N}, 111.6^{\circ} \mathrm{W}\right)$, portraying propagating gravity waves. The periods, horizontal wavelengths, phase speeds and propagation azimuths are a $13.5 \mathrm{~min}, 26 \mathrm{~km}, 32 \mathrm{~m} / \mathrm{s}$ and $85^{\circ}$, and $\mathbf{b} 5.2 \mathrm{~min}$,
$15 \mathrm{~km}, 48 \mathrm{~m} / \mathrm{s}$ and $5^{\circ}$, respectively. The white lines perpendicular to the wave fronts and passing through the zenith indicate the scans shown in Fig. 2. For a detailed explanation, see the text 
are made using exposure times ranging from $15 \mathrm{~s}$ for the near-infrared $\mathrm{OH}$ to $90 \mathrm{~s}$ for the fainter $\mathrm{OI}$ and $\mathrm{Na}$ emissions (Taylor et al., 1995a). Figure 1a shows a series of waves oriented approximately in the $\mathrm{N}-\mathrm{S}$ direction and exhibiting a horizontal wavelength of $26 \mathrm{~km}$ and an observed phase speed of $32 \mathrm{~m} / \mathrm{s}$ (the observed period is $13.5 \mathrm{~min}$ ). The data were obtained on 27 May 1995. In comparison, Fig. 1b shows a series of smaller-scale waves (horizontal wavelength $15 \mathrm{~km}$, phase speed $48 \mathrm{~m} /$ $\mathrm{s}$, indicating an observed period of only $5.2 \mathrm{~min}$ ) imaged on 24 June 1995. In both cases the wave patterns are spatially coherent and horizontally extensive, occupying most of the image area. Intensity scans across a selected region of each of these wave patterns, indicated by the white lines in Fig. 1, are shown in Fig. 2. Prior to scanning each image was "flat-fielded" to minimize the effects of line-of-sight enhancement of the airglow signal and lens vignetting on the measurement. Both traces show a well-defined intensity modulation characteristic of the gravity wave pattern and each indicates an induced contrast ratio $\left(I_{\max }-I_{\min }\right) / I_{a v}$ of approximately $5-10 \%$ which is typical of many wave patterns imaged in the nightglow emissions.

\section{Inversion method}

The most common inversion methods used in satellite or auroral tomography are recursive algorithms like MART, ART or SIRT (see e.g. Austen et al., 1988; Raymund et al., 1990; Andreeva et al., 1992). In this sort of approach one starts from some initial distribution and continues the iteration until the stop criterion is
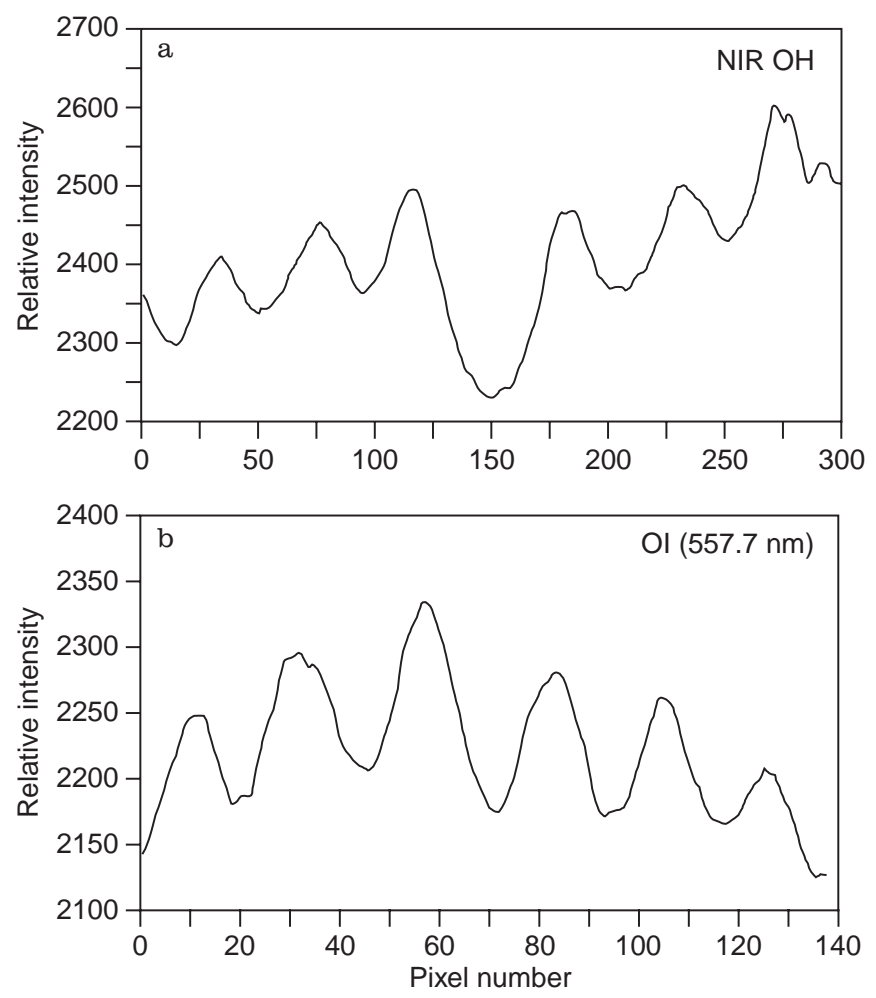

Fig. 2. Scans along the white lines across the airglow images in Fig. 1 met. The stop criterion and especially the start distribution affect the inversion result and choosing them is a problem itself. A clarifying viewpoint to the matter is to understand that the choice of the start distribution is equivalent to adopting some a priori information which is then utilized by the solver. This a priori information determines which one of the infinite number of possible solutions satisfying the experimental data will be given as the final result.

A less frequently applied tomographic method is based on stochastic inversion, which has been utilized in satellite tomography e.g. by Fremouw et al., (1992) and Markkanen et al., (1995). This approach gives the most probable solution satisfying the measurements, normally within some constraints which can be interpreted as an input of a priori information. In this paper, stochastic inversion is applied in airglow tomography using the formulation by Markkanen et al., (1995). Since the method was described in great detail by Nygrén et al., (1997b), it is only briefly outlined here.

We assume that the airglow luminosity is observed by a chain of ground-based imagers, and we only consider the luminosity from the vertical plane above the camera chain. Hence the data will consist of scans across the airglow images such as those in Fig. 2, one for each camera, and the result will be an image of volume emission rate on this plane. Thus we restrict ourselves to a two-dimensional inversion problem. After appropriate corrections, the signal from every pixel on the airglow image scans is equal to the integral of volume emission rate along a given line in the image plane. The signal also contains random noise and a possible camera offset value.

The image plane is divided into grid elements, which, due to the curvature of the Earth, are not exactly rectangular but annular-shaped. The grid extends horizontally over the region seen at least by one of the cameras. The programme package allows the use of different element sizes in different parts of the plane. In practice, small elements are used in the centre region where observations from more than a single camera are available, and large elements at the edges. Large elements are also used at low altitudes where the volume emission rate is expected to be zero. With this choice the size of the inversion problem can be effectively reduced.

All measurements from the pixels on the image scans are collected to a single column vector denoted by $\mathbf{m}$. The volume emission rates at the grid points as well as the camera offsets are the unknowns, which are collected to a column vector $\mathbf{x}$. Using bilinear interpolation within the grid, the relationship between measurements and unknowns will be linear and can be written in the form

$\mathbf{m}=\mathbf{A}_{\mathbf{m}} \cdot \mathbf{x}+\varepsilon_{\mathbf{m}}$,

where $\varepsilon_{\mathbf{m}}$ is a column vector of the measurement errors. Here $\mathbf{A}_{\mathbf{m}}$ is a matrix which depends on the choice of the grid, the camera locations and the direction of observation of each component of $\mathbf{m}$.

In stochastic inversion both $\mathbf{m}, \mathbf{x}$ and $\varepsilon_{\mathbf{m}}$ are treated as random variables, and the solution of the inversion 
problem gives the most probable value of $\mathbf{x}$. Without regularization, however, the solution is unstable. In the present formalism, stability is achieved by treating the point-to-point changes of the volume emission rate as random variables and controlling the size of these changes by respective variances. If all point-to-point changes of the unknowns both in vertical and horizontal directions are arranged as a column vector $\varepsilon_{\mathbf{r}}$, we can formally write

$\mathbf{0}=\mathbf{A}_{\mathbf{r}} \cdot \mathbf{x}+\varepsilon_{\mathbf{r}}$,

where $\mathbf{A}_{\mathbf{r}}$ is a matrix with elements $+1,-1$ and 0 in appropriate places so that a correct difference of unknowns will appear on each row. When both $\varepsilon_{\mathbf{m}}$ and $\varepsilon_{\mathbf{r}}$ are treated as Gaussian random variables with zero mean, the solution of the inversion problem can be written as

$\mathbf{x}_{\mathbf{0}}=\left(\mathbf{A}_{\mathbf{m}}{ }^{T} \cdot \boldsymbol{\Sigma}_{\mathbf{m}}^{-1} \cdot \mathbf{A}_{\mathbf{m}}+\mathbf{A}_{\mathbf{r}}^{T} \cdot \mathbf{\Sigma}_{\mathbf{r}}^{-1} \cdot \mathbf{A}_{\mathbf{r}}\right)^{-1} \cdot \mathbf{A}_{\mathbf{m}}^{T} \cdot \boldsymbol{\Sigma}_{\mathbf{m}}^{-1} \cdot \mathbf{m}$.

Here $\boldsymbol{\Sigma}_{\mathbf{m}}$ and $\boldsymbol{\Sigma}_{\mathbf{r}}$ are the covariance matrices of $\varepsilon_{\mathbf{m}}$ and $\varepsilon_{\mathbf{r}}$, respectively. The stability of this solution is controlled by the covariance matrix $\Sigma_{\mathbf{r}}$. If the variance corresponding to a given pair of unknowns is zero within $\Sigma_{\mathbf{r}}$, no point-to-point change is possible. If the variance is small, only a small change is likely, but if it is large, also a great point-to-point change may (although does not necessarily) take place.

As demonstrated by Saksman et al. (1997), this sort of inversion problem has an infinite number of solutions. In our method the choice of the final solution from this set is determined by the regularization variances, which are selected by the user. In this way the user can take advantage of possible a priori information available from other sources, e.g. models or independent measurements. One should notice that in recursive algorithms the equivalent a priori information is hidden in start distributions and stop criteria; in stochastic inversion the inclusion of a priori information can only be made in a more controlled way.

\section{Inversion of simulated data}

Since wave structures are often observed in airglow images, different types of wave models were constructed for data simulation. Figure 3 a shows a simple model wave packet with tilted wave fronts at $85 \mathrm{~km}$ altitude, representing the effect of a gravity wave on airglow
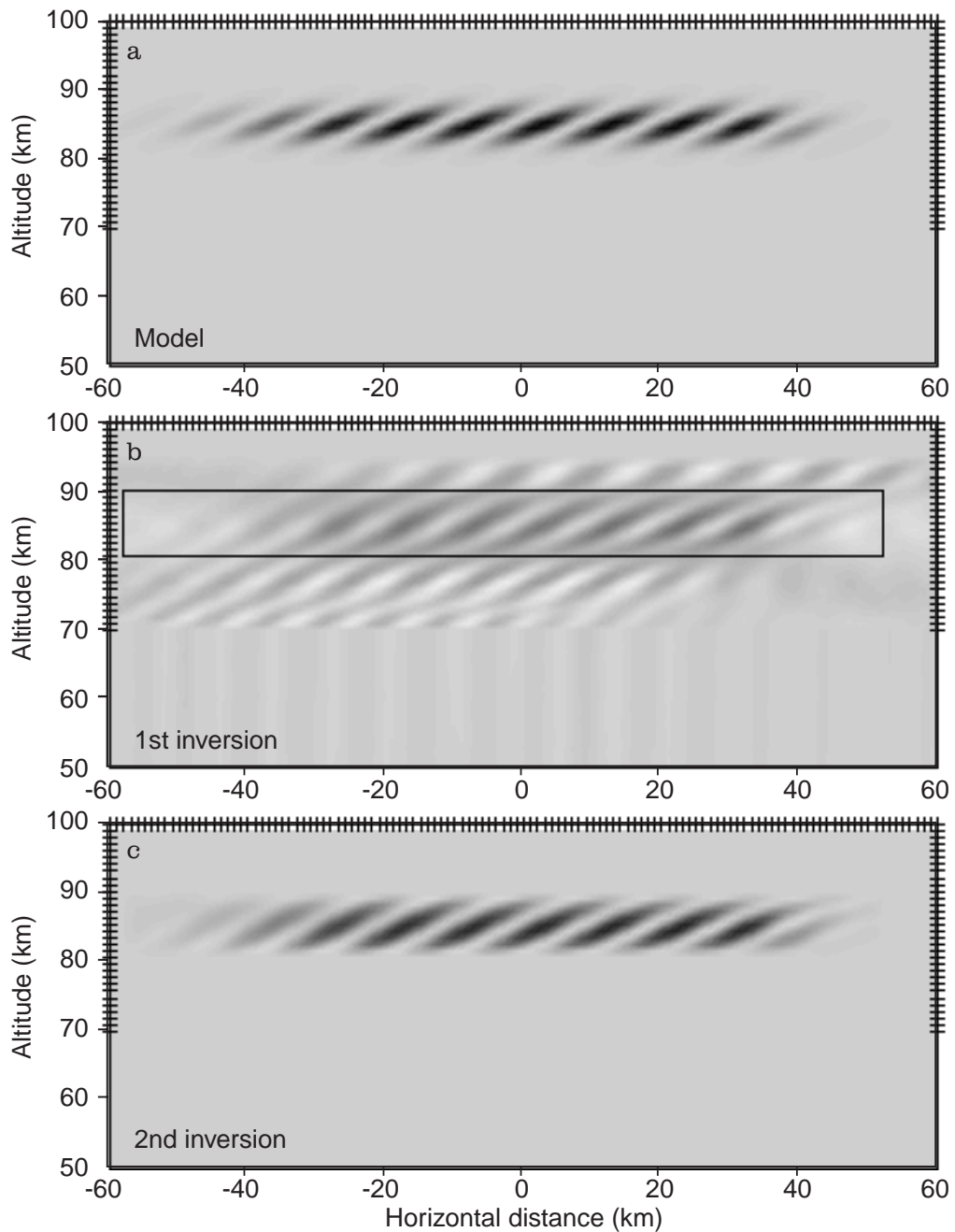
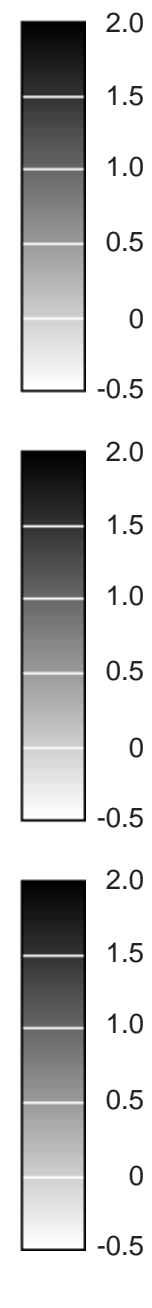

Fig. 3. a Model of airglow volume emission rate indicating a short-scale gravity wave. The model contains a Gaussian vertical profile with a scale length of $2.5 \mathrm{~km}$, centred at 85 $\mathrm{km}$ altitude and modulated by a wave with horizontal and vertical wavelengths of 10 and $5 \mathrm{~km}$, respectively. Both ends of the model are tapered horizontally by Gaussian functions. b Results of the first inversion of the data in Fig. 4. The regularization box is indicated by a black line (see the text). c Results of the second inversion of the data in Fig. 4, carried out using constant non-zero regularization variances inside the box in panel $b$ and zero variances elsewhere 
luminosity. The horizontal and vertical wavelengths are 10 and $5 \mathrm{~km}$, respectively, and the vertical profile of the wave amplitude is Gaussian with a scale height of 2.5 $\mathrm{km}$. One should notice that the same tilt of the wave front is obtained if the horizontal and vertical wavelengths are multiplied by the same factor. Therefore, a model with a $20-\mathrm{km}$ horizontal and a $10-\mathrm{km}$ vertical wavelength would look much like that in Fig. 3a, only the separation of the wave fronts would be doubled in horizontal direction.

Five cameras at separations of $100 \mathrm{~km}$ were assumed on the ground level below this model in a chain extending from -200 to $200 \mathrm{~km}$. Simulated data were calculated for each camera using 600 directions above elevation angles of $10^{\circ}$. Gaussian random noise with a standard deviation of one unit was added to the data. Central parts of these data are shown in Fig. 4, where the ground projection of ray crossing of $100 \mathrm{~km}$ height is used as the horizontal axis instead of the elevation angle. It is observed that clear oscillations are visible in signals from the cameras at -200 and $-100 \mathrm{~km}$, whereas the cameras at 100 and $200 \mathrm{~km}$ only see a wide unstructured region of luminosity. This is because, in the latter case, the wave fronts are seen more or less from a perpendicular direction so that the individual wave fronts cannot

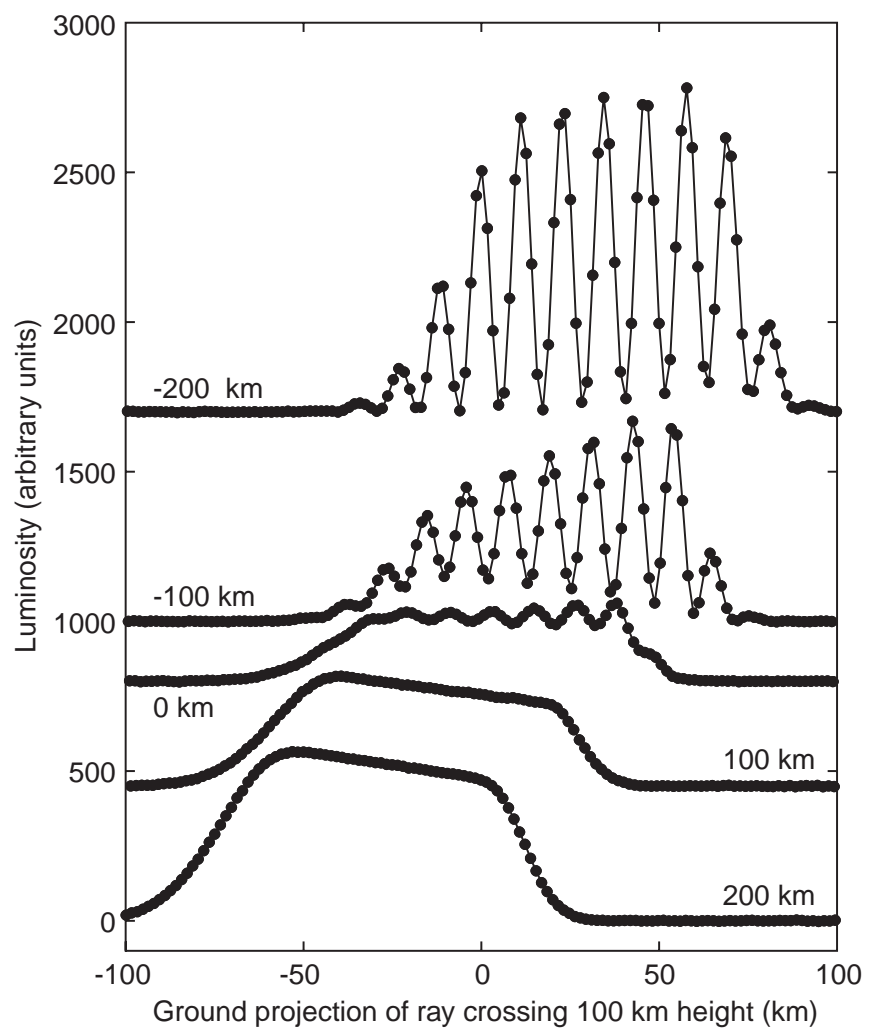

Fig. 4. Simulated airglow data for five imagers located at -200 , $-100,0,100$ and $200 \mathrm{~km}$ on the ground level below the model in Fig. 3a. The number of data points for each imager is 600 at equal steps of the elevation angle above $10^{\circ}$. Gaussian random noise with a standard deviation of 1 unit has been added to the data. The zero level of the luminosity scale refers to the imager at $200 \mathrm{~km}$; the other curves are drawn starting from different zero levels in order to avoid overlapping be distinguished. Similar cases are encountered in satellite radiotomography when travelling ionospheric disturbances are propagating in the F-layer altitudes (e.g. Pryse et al., 1995; Saksman et al., 1997).

The inversion is carried out in two stages. In the first stage, a constant regularization variance is used in a wide region within the grid. The results of the first inversion are then used in choosing new regularization variances, which are applied in the second inversion leading to the final result.

The result of the first inversion of the data in Fig. 4 is shown in Fig. 3b (the meaning of the box inside the panel will be explained below). Here the tick marks on the top and right-hand side of the panel indicate the applied grid with an element size of $1 \mathrm{~km}$ above $70 \mathrm{~km}$ altitude both in horizontal and vertical direction. Below this height, the next grid points are on the ground level, so that the heights of the bottom elements of the grid are $70 \mathrm{~km}$. Large horizontal element sizes are used at the edges of the grid, but they are not visible in the figure. Constant non-zero horizontal and vertical regularization variances are chosen within the altitude range 70-95 $\mathrm{km}$ and zero variances outside it. In addition, the solution is put to zero both on the ground level and at $100 \mathrm{~km}$ height. Together with the choice of variances, this means that the solution will be zero outside the 70 $95 \mathrm{~km}$ height region.

The result indicates that the first inversion is capable of reproducing a correct number of tilted wave fronts in correct horizontal positions. The altitude of maximum amplitude is also the same as in the model. The wave fronts, however, extend well beyond the range of the model in vertical direction. However, a more detailed inspection of the reconstruction reveals that the character of the solution within the model region differs from that outside it. This is seen in Fig. 5, where two horizontal profiles of the first inversion are shown. The profile from $86.5 \mathrm{~km}$ altitude shows a set of strong positive peaks and only small negatives, whereas the solution at $77.5 \mathrm{~km}$ oscillates more or less around zero. The latter profile comes from an altitude where the model is practically zero. Hence it seems possible to use this property of the first inversion result to line out the region where the airglow luminosity has a significant amplitude. Both horizontal and vertical profiles of the first inversion result were investigated in order to find the boundary where the solution changes from mainly positive to the unrealistic type containing also strong negatives. This boundary is indicated by the box in Fig. 3b.

After the region of airglow emission is found by the foregoing method, a second inversion is carried out using new regularization variances. Non-zero constant variances are now used only inside the outlined region. Elsewhere the variances are put to zero with the result that the solution will also be zero there. Therefore, a non-zero inversion result will be obtained only within the region deduced from the result of the first inversion.

The result of the second inversion is shown in Fig. 3c. The similarity of this reconstruction and the model is remarkable; both the peak altitude, the vertical extent, and the number and orientation of the wave fronts is 


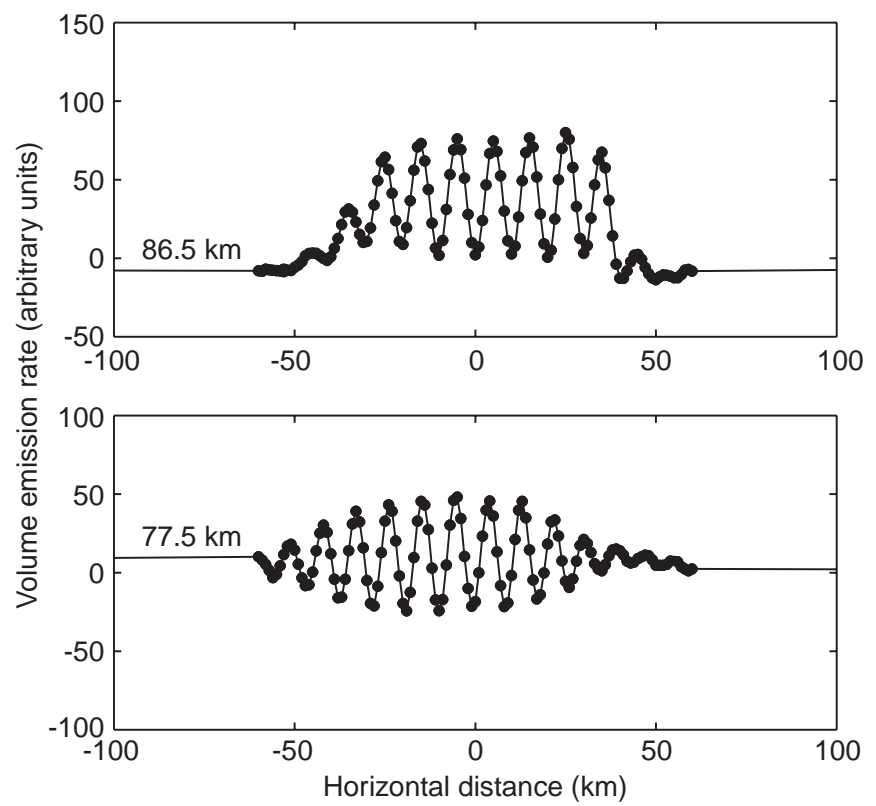

Fig. 5. Horizontal scans of the first inversion in Fig. $3 b$ from altitudes 77.5 and $86.5 \mathrm{~km}$ correct. A more detailed comparison reveals minor differences, however. For instance, the peak values of the inversion result are smaller than those of the model, and small negatives appear at some parts of the troughs of the wave.

As a second example, another wave packet centred at $80 \mathrm{~km}$ altitude is added to the preceding model. Its amplitude is the same as that of the first wave, its horizontal and vertical wavelengths are 7.5 and $5 \mathrm{~km}$, respectively, and the vertical scale height of the Gaussian amplitude is $1.5 \mathrm{~km}$. This model is plotted in Fig. $6 \mathrm{a}$. Data for the same set of camera sites were then calculated as described above. Figure. $6 \mathrm{~b}$ shows the result of the first inversion and the boundary found by investigating the horizontal and vertical profiles. Finally, the result of the second inversion, carried out using non-zero regularization variances within the boundary, is portrayed in Fig. 6c. Even in this case the model is quite well reproduced by the second inversion, although minor discrepancies are revealed in a detailed comparison.

A more complicated example is presented in Fig. 7. Here a third wave packet with the same parameters as
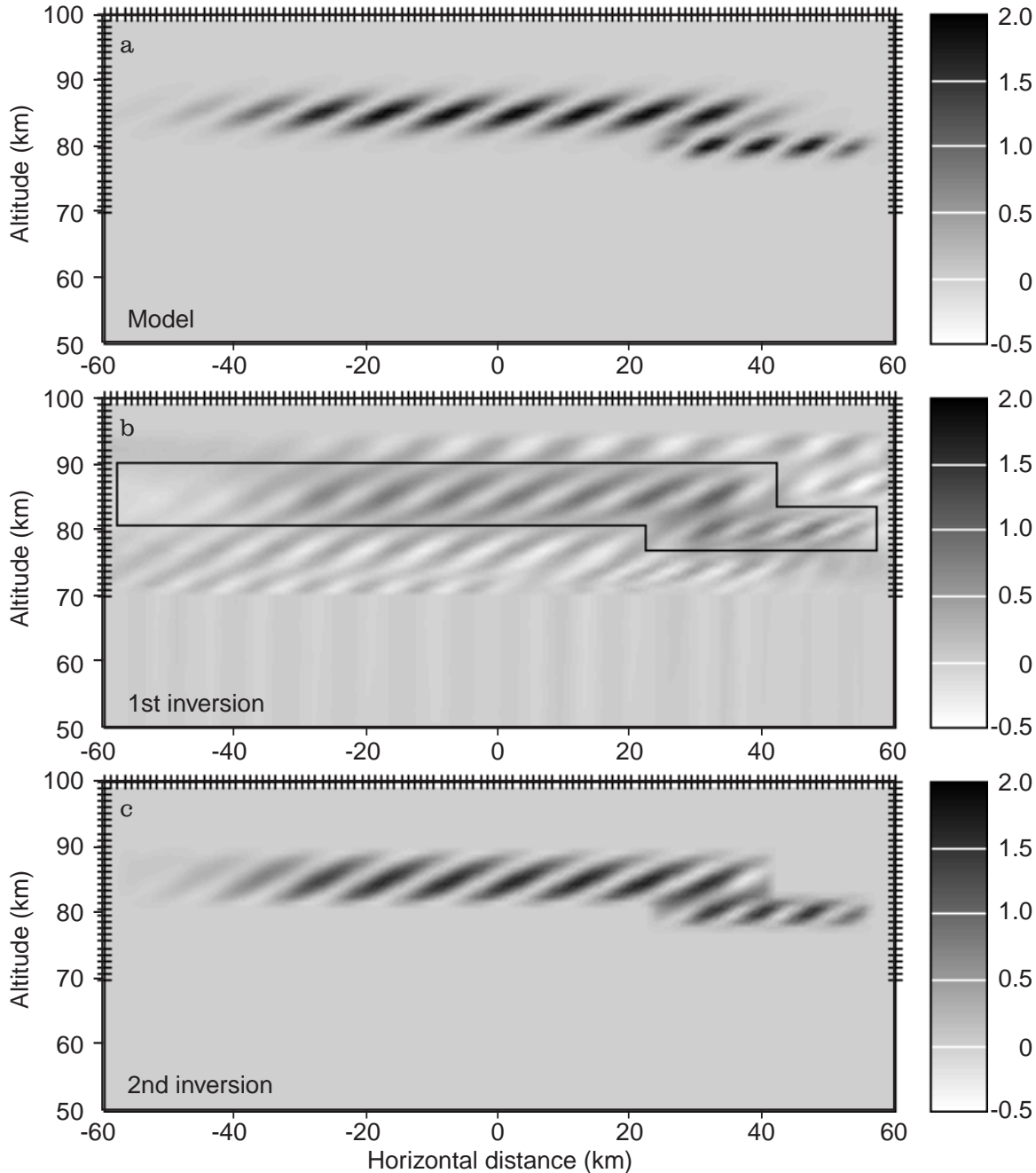

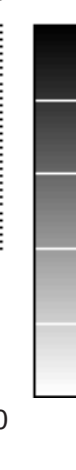

Fig. 6. Same as Fig. 3, with a second wave packet on the right-hand side of the model. The peak altitude of the second wave is $80 \mathrm{~km}$, the vertical scale height of the amplitude is 1.5 $\mathrm{km}$, and the horizontal and vertical wavelengths are 7.5 and $5 \mathrm{~km}$, respectively 

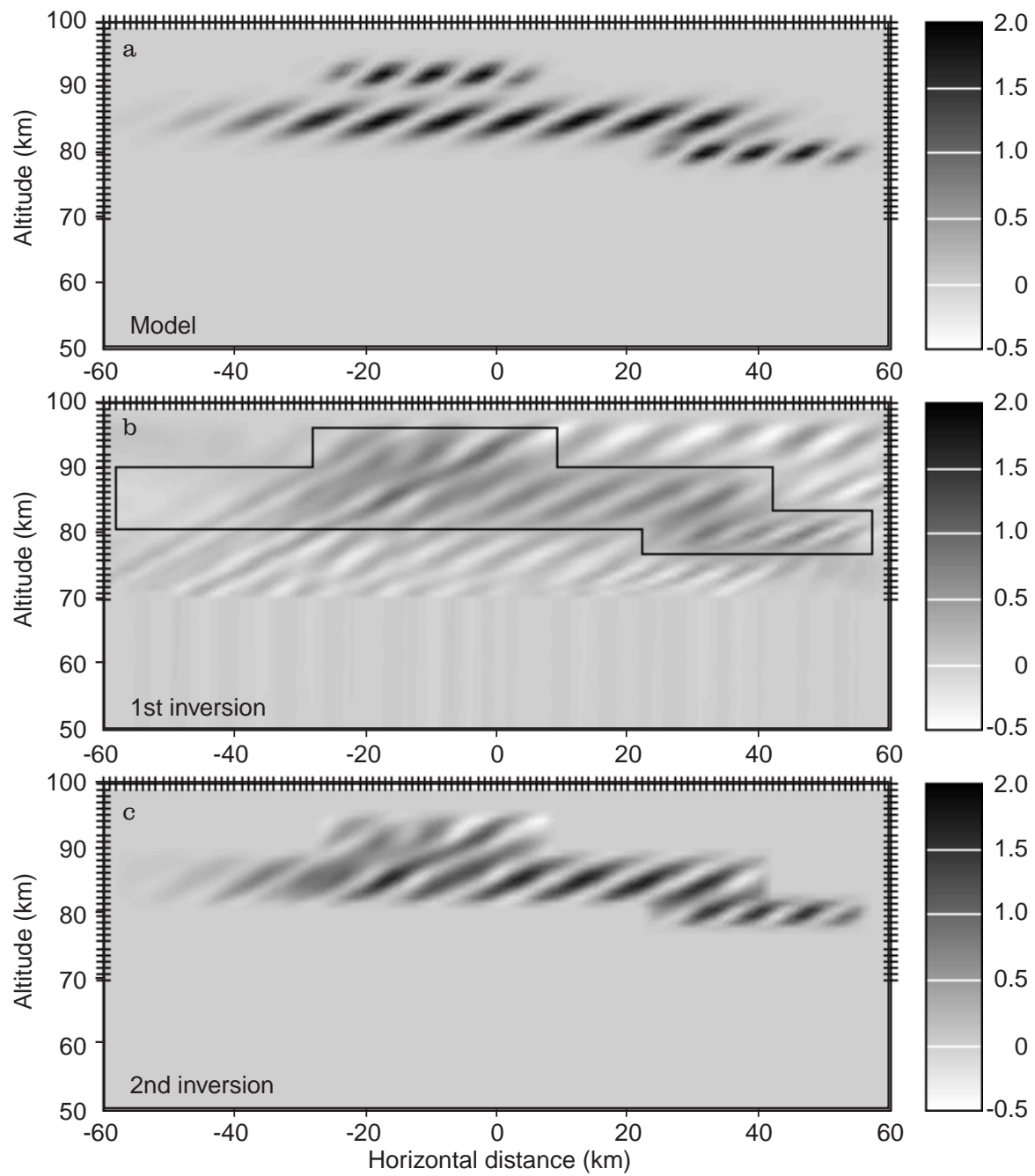

Fig. 7. Same as Fig. 6, with a third wave packet added on the top of the model. The wavelengths and scale height of this wave are the same as those of the second wave packet, but its peak altitude is $92 \mathrm{~km}$

the second one in Fig. 6 is added at the altitude of $92 \mathrm{~km}$. Simulated data were calculated with the same camera chain as above. This case is more demanding than the other two, because the wave at a lower altitude is shadowing the third packet. The results of the first and second inversion are shown in Fig. 6b and c, respectively. The final result indicates that the number of reconstructed wave fronts in the topmost packet is correct. The altitude of the wave fronts is not constant, however, but the second and fifth front from the left are shifted downwards. Some deformation of the lower wave is also observed.

The reason why the inversion result in Fig. 7 is worse than in the other two examples is obvious: the topmost wave packet is hiding behind the lower ones as seen by all cameras in the chain. Depending on the relative locations of the wave fronts in the two packets, a given front in the topmost wave may or may not be visible well enough to be properly reconstructed. Examples of both cases are present in Fig. 7. For a better reconstruction, one should include lower-elevation rays in the experimental setup. In order to achieve this, an attempt was made to add two cameras in the system at -300 and 300 $\mathrm{km}$. Although some improvement was gained in the results, clear vertical shifts of the wave fronts were still present in the reconstruction.

Tests were also made to invert data obtained from a chain of three cameras, extending from -200 to $200 \mathrm{~km}$ on the ground level. The simple wave model in Fig. 3 was used in these tests. It turned out that the first inversion clearly revealed the wave structure, but the inversion results showed less clearly the boundaries for determining the regularization variances for the second inversion. If the same boundaries were used as before, the results, although not quite as good as for five cameras, were still in a reasonable agreement with the original model. Thus it seems that a chain of three cameras might still be acceptable, although difficulties may arise in determining the regularization.

Another check was to investigate the effect of making the camera chain shorter. This test was carried out assuming five cameras distributed at equal separations from -100 to $100 \mathrm{~km}$. When the wave model in Fig. 3 was used, the first reconstruction was able to reveal the wave structure, but finding the regularization boundaries for the second reconstruction was problematic even in this case. If the same procedure as in Fig. 3 was applied, the resulting layer was too thick. The explana- 
tion is that, in a short chain, the model is visible to all cameras only at high elevation angles, and therefore the data contain less information on the vertical profile than data from a longer chain do.

In all the foregoing examples the thickness of the luminous layer is of the order of the vertical wavelength. It is of some interest to see what happens if the layer is thicker than the vertical wavelength. This was investigated using the same set of cameras as in Fig. 3 and expanding the model in vertical dimension. The results are plotted in Fig. 8, where the top panel shows the model extending approximately between 77 and $93 \mathrm{~km}$ in height. The height span of the regularization box determined from the first inversion is now wider than in Fig. 3. Although there are minor distortions in individual wave fronts, the model wave is clearly reproduced by the second inversion with correct vertical and horizontal wavelengths. The fact that the reconstructed layer is somewhat too thick indicates uncertainties associated with the choice of the regularization box. The success in the reconstruction can be understood when noticing that the cameras at -100 and $-200 \mathrm{~km}$ have a view more or less in the direction of the wave fronts. Thus they are able to resolve the individual fronts even when the layer is thicker than the vertical wavelength. Then the inversion routine has a sufficient information on the wave structure for reproducing it with a reasonable accuracy.

\section{Discussion}

Airglow observations provide a method which has a great potential power for investigating the properties of atmospheric gravity waves close to the mesopause altitude. This is because the airglow luminosity acts as a tracer of density enhancements associated with the wave propagation. Until now, it has been possible to determine the horizontal wavelength from individual airglow images, the direction of wave propagation, the period and the horizontal phase velocity from a set of successive images, and the altitude of the luminosity by triangulation from images taken at separate sites. However, airglow data obtained simultaneously at several sites contain even more information which can be extracted by means of tomographic inversion. More specifically, tomographic analysis allows the determination of the vertical wavelength and the vertical phase
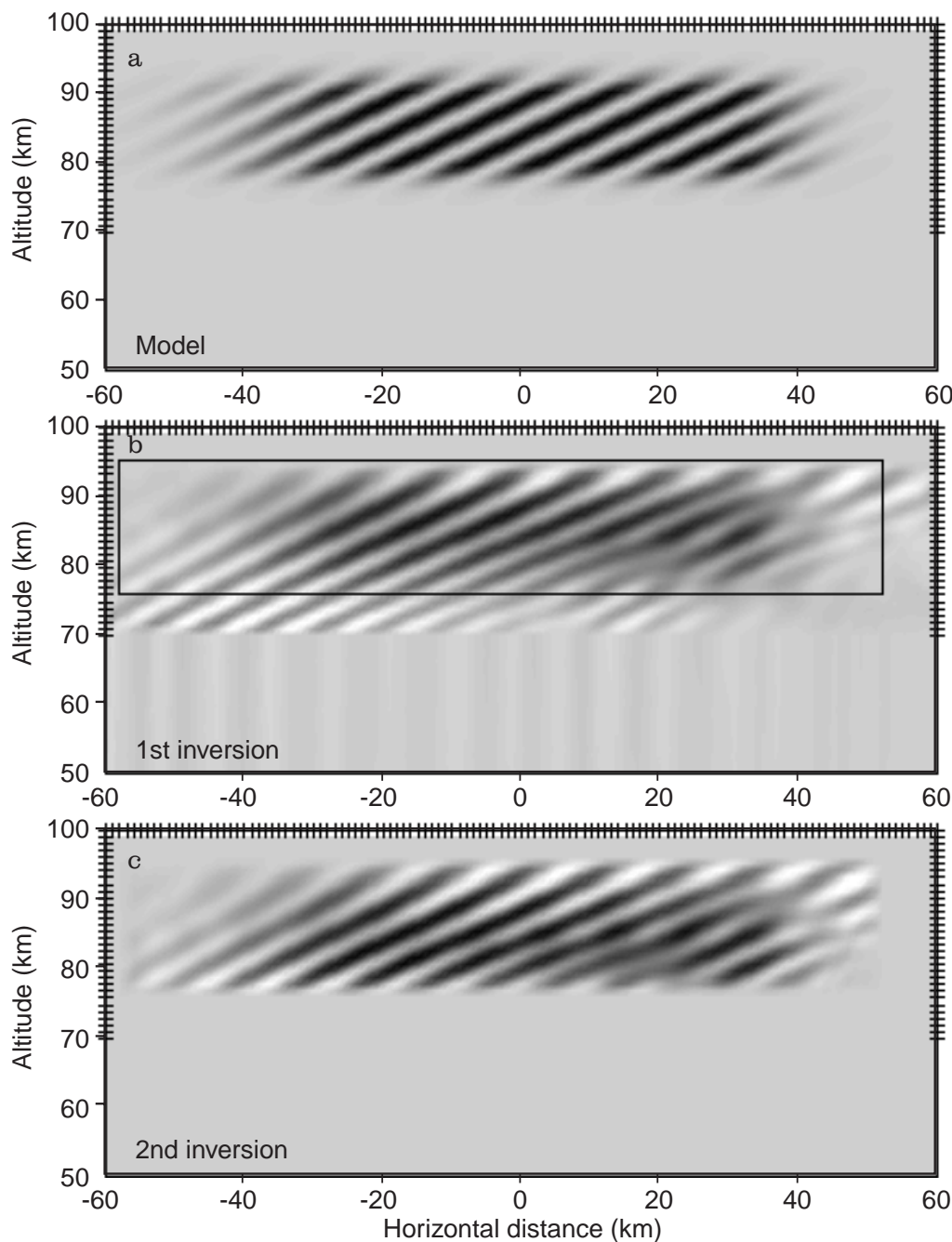
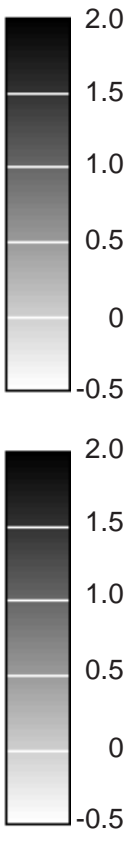

2.0

.5

1.0

1.5

1.0

0.5

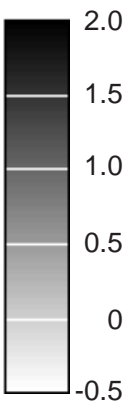

Fig. 8. Same as Fig. 3 with a thicker model layer. Here the wave amplitude is constant between 80 and $90 \mathrm{~km}$ and decreases with Gaussian scale heights of $2.5 \mathrm{~km}$ around this region. The wavelengths are the same as in Fig. 3 
velocity so that a full study of the dispersive properties of the gravity waves will become possible.

When designing a proper experimental setup for airglow tomography, it is essential to know the number of imagers needed in tomographic work as well as their optimal separations. The simulations carried out in the present paper are planned to act as guidelines for such a goal. It seems clear that at least three cameras are needed and they should be installed in a chain with separations of the order of $100 \mathrm{~km}$ for investigation of small-scale waves. Additional imagers at longer distances at both ends of the chain would be highly desirable for studying large-scale phenomena. One should point out that the data encountered in true measurements may be much more variable than that produced in data simulations, and therefore experience with real data may alter the ultimate design of an imager chain.

The tomographic inversions in this paper were carried out without actually making use of any a priori information on the height and the thickness of the luminous layer. Even the regularization variances, which provide a means of importing a priori information to the solver, were chosen using only the properties of the data. In reality we have pretty good a priori information on the altitude range of a given emission. This may help in choosing the regularization box and therefore we do not necessarily have to rely only on the first inversion as in the present simulations. Another point making the reconstructions easier is that different emissions actually come from different heights and they are separated by filters. Therefore we do not really have to analyse such complicated cases as in Figs. 6 and 7, but rather our inversion problems will be more like those in Figs. 3 and 8. The complicated cases were included in this paper in order to demonstrate both the limitations and the power of the tomographic method.

An interesting result in the foregoing simulations is that layers even thicker than a couple of vertical wavelengths can be successfully reconstructed. This seems to be in contradiction to what one might assume at first sight, since the wave effects are expected to be smoothed out by integration through the layer. Even in this case, however, it is possible to see through the layer when looking at the direction of the tilted wave fronts. Therefore the tomographic inversion becomes possible, if the data contain observations from such directions. The orientation of the wave fronts may affect the result; if the orientation of the wave fronts is too close to horizontal, the data may not contain measurements from appropriate directions. Here it is worth pointing out that gravity waves have also been observed at Fregion heights by means of satellite tomography. These observations clearly demonstrate how waves can be reconstructed in regions where rays parallel to the wave fronts contribute to the data, but in regions where all rays from the satellite to the receivers are nearly perpendicular, no waves appear in the inversion results (Markkanen et al., 1995; Saksman et al., 1997).

A conclusion taken from the preceding discussion is that the most important factor for the success in the reconstruction of gravity waves is the orientation of the wave fronts rather than the wavelength or the layer thickness. Therefore, although our examples contain only a limited choice of small-scale waves, a similar success in tomographic inversion is also expected at longer wavelengths, as long as the orientation of the wave fronts is not too horizontal. If both the vertical and horizontal wavelengths in the above examples are multiplied by the same factor, only the separation of the wave fronts change but their orientation remains the same. Therefore it is obvious that no difficulties should appear in reconstructing waves with longer vertical wavelengths, e.g. $10-20 \mathrm{~km}$.

A major limitation of the tomographic method in its present form is that it is restricted to one-dimensional data sets and two-dimensional reconstructions. Since the airglow images give two-dimensional data, the method only makes use of a small fraction of the available information. For a more complete utilization of the observations, three-dimensional tomographic inversion should be carried out. In principle, a three-dimensional tomographic package could be created using the same inversion kernel, but programming the three-dimensional geometry would be a major task. Instead of a chain of imagers, one should also have a two-dimensional network. Moving to three dimensions would make the inversion problem essentially bigger so that the demand of CPU time would greatly increase and, in practice, such spatial resolutions as in two dimensions could hardly be achieved.

As a final comment, we should remember that the data collected in an experiment discussed in the present work are similarly defective as that in satellite radio tomography: they are obtained as a result of a poor sampling of the Radon transform of the unknown function. This means that the data cannot be inverted in a unique way (not even in the absence of noise), and therefore the results may be subject to uncertainties (see e.g. Markkanen et al., 1995; Saksman et al., 1997; Nygrén et al., 1997b). Hence there is no way to create a mathematical criterion for judging the validity of the results, but one has to rely on a critical evaluation of the result. Because of the thinness of the airglow layer, the uncertainties are probably weaker in airglow tomography than in satellite tomography.

Acknowledgements. The authors are grateful to M.H. Rees for pointing out the airglow tomography work done on Atmospheric Explorer data. The Bear Lake image measurements were supported by a grant from the National Science Foundation, USA, number ATM-9525815. Support from the Academy of Finland is also gratefully acknowledged.

Topical Editor D. Alcaydé thanks E. Pryse and S.B. Mende for their help in evaluating this paper.

\section{References}

Abreu, V. J., and J. H. Yee, Diurnal and seasonal variation of the nighttime $\mathrm{OH}$ (8-3) emission at low altitudes, J. Geophys. Res., 94, 11949-11957, 1989.

Andreeva, E. S., V. E. Kunitsyn, and E. D. Tereshchenko, Phasedifference radiotomography of the ionosphere, Ann. Geophysicae, 10, 849-855, 1992. 
Aso, T., and M. Ejiri, CAT - a method for the determination of aurora luminosity structures, in The 19th Annual European meeting on atmospheric studies by optical methods, August 1014, 1992, Kiruna, Sweden, IRF Scientific Report 209, 249-254, 1992.

Aso, T., T. Hashimoto, M. Abe, T. Ono, and M. Ejiri, On the analysis of aurora stereo observations, J. Geomagn. Geoelect., 42, 579-595, 1990.

Austen, J. R., S. J. Franke, C. H. Liu, and K. C. Yeh, Application of computerized tomography techniques to ionospheric research, in Radio beacon contribution to the study of ionisation and dynamics of the ionosphere and corrections to geodesy, Ed. A. Tauriainen, Part 1, 25-35, 1986.

Austen, J. R., S. J. Franke, and C. H. Liu, Ionospheric imaging using computerized tomography, Radio Sci., 23, 299-307, 1988.

Baker, D. J., and A. T. Stair, Jr., Rocket measurements of the altitude distributions of the hydroxyl airglow, Phys. Scr., 37, 611-622, 1988.

Clairemidi, J., M. Herse, and G. Moreels, Bi-dimensional observations of waves near the mesopause at auroral latitudes, Planet. Space Sci., 33, 1013-1022, 1985.

Fremouw, E. J., J. A. Secan, and B. M. Howe, Application of stochastic inverse theory to ionospheric tomography, Radio Sci., 27, 721-732, 1992.

Fritts, D. C., and R. A. Vincent, Mesospheric momentum flux studies at Adelaide, Australia: observations and gravity wavetidal interaction model, J. Atmos. Sci., 44, 605-619, 1987.

Kunitsyn, V. E., and E. D. Tereshchenko, Radio tomography of the ionosphere, IEEE Antennas Propag. Mag., 34(5), 22-32, 1992.

Markkanen, M., M. Lehtinen, T. Nygrén, J. Pirttilä, P. Henelius, E. Vilenius, E. D. Tereshchenko, and B. Z. Khudukon, Bayesian approach to satellite radiotomography with applications in the Scandinavian sector, Ann. Geophysicae, 13, 1277-1287, 1995.

McDade, I. C., and E. J. Llewellyn, Inversion techniques for recovering two-dimensional distributions of auroral emission rates from tomographic rocket photometer measurements, Can. J. Phys., 69, 1059-1068, 1991.

McDade, I. C., N. D. Lloyd, and E. J. Llewellyn, A rocket tomography measurement of the $\mathrm{N}_{2}^{+} 3914 \AA$ emission rates within an auroral arc, Planet. Space Sci., 39, 895-906, 1991.

Mitchell, C. N, L. Kersley, J. A. T. Heaton, and S. E. Pryse, Determination of vertical electron-density profile in ionospheric tomography: experimental results, Ann. Geophysicae, 15, 747752, 1997.

Moreels, G., and M. Herse, Photographic evidence of waves around the $85 \mathrm{~km}$ level, Planet. Space Sci., 25, 265-273, 1977.

Nygrén, T., M. Markkanen, M. Lehtinen, and K. Kaila, Application of stochastic inversion in auroral tomography, Ann. Geophysicae, 14, 1124-1133, 1996a.

Nygrén, T., M. Markkanen, M. Lehtinen, E. D. Tereshchenko, B. Z. Khudukon, O. V. Evstafiev, and P. Pollari, Comparison of Fregion electron density observations by satellite radio tomography and incoherent scatter methods, Ann. Geophysicae, 14, 1422-1429, 1996b.

Nygrén, T., M. Markkanen, M. Lehtinen, and K. Kaila, Tomographic inversion of auroral camera or photometer observations, Adv. Space Phys., 19, 639-642, 1997a.
Nygrén, T., M. Markkanen, M. Lehtinen, E. D. Tereshchenko, and B. Z. Khudukon, Stochastic inversion in ionospheric radiotomography, Radio Sci., 32, 2359-2372, 1997b.

Peterson, A. W., and L. M. Kieffaber, Photographic parallax heights of infrared airglow structures, Nature, 244, 92-93, 1973.

Pryse, S. E., and L. Kersley, A preliminary experimental test of ionospheric tomography, J. Atmos. Terr. Phys., 54, 1007-1012, 1992.

Pryse, S. E., L. Kersley, D. L. Rice, C. D. Russell, and I. K. Walker, Tomographic imaging of the mid-latitude trough, Ann. Geophysicae, 11, 144-149, 1993.

Pryse, S. E., C. N. Mitchell, J. A. T. Heaton, and L. Kersley, Travelling ionospheric disturbances imaged by tomographic techniques, Ann. Geophysicae, 13, 1325-1330, 1995.

Raymund, T. D., J. R. Austen, S. J. Franke, C. H. Liu, J. A. Klobuchar, and J. Stalker, Application of computerized tomography to the investigation of ionospheric structures, Radio Sci., 25, 771-789, 1990.

Saksman, E., T. Nygrén, and M. Markkanen, Ionospheric structures invisible in satellite radiotomography, Radio Sci., 32, 605616, 1997.

Solomon, S. C., P. B. Hays, and V. J. Abreu, Tomographic inversion of satellite photometry, Appl. Opt., 23, 3409-3414, 1984.

Solomon, S. C., P. B. Hays, and V. J. Abreu, Tomographic inversion of satellite photometry. Part 2, Appl. Opt., 24, 41344140, 1985.

Solomon, S. C., P. B. Hays, and V. J. Abreu, The auroral $6300 \AA$ emission: observations and modeling, J. Geophys. Res., 93, 9867-9882, 1988

Swenson, G. R., and S. B. Mende, $\mathrm{OH}$ emission and gravity waves (including a breaking wave) in all-sky imagery from Bear Lake, UT., Geophys. Res. Lett., 21, 2239-2242, 1994.

Taylor, M. J., M. A. Hapgood, and P. Rothwell, Observations of gravity wave propagation in the $\mathrm{OI}(557.7 \mathrm{~nm}), \mathrm{Na}(589.2 \mathrm{~nm})$ and the near infra-red $\mathrm{OH}$ nightglow emissions, Planet. Space Sci., 35, 413-427, 1987.

Taylor, M. J., M. B. Bishop, and V. Taylor, All-sky measurements of short period waves imaged in the OI $(557.7 \mathrm{~nm}), \mathrm{Na}(589.2$ $\mathrm{nm})$ and near infrared $\mathrm{OH}$ and $\mathrm{O} 2(0,1)$ nightglow emissions during the ALOHA-93 campaign, Geophys. Res. Lett., 22, 2833-2836, 1995a.

Taylor, M. J., G. R. Swenson, and V. Taylor, Height measurements of $\mathrm{OI}(557.7 \mathrm{~nm})$ gravity wave structure over the Hawaiian Islands during ALOHA-93, Geophys. Res. Lett., 22, 2881-2884, 1995b.

Taylor, M. J., W. R. Pendleton, Jr., S. Clark, H. Takahashi, D. Gobbi, and R.A. Goldberg, Image measurements of short-period gravity waves at equatorial latitudes, J. Geophys. Res., in press 1998.

Vallance Jones, A., R. L. Gattinger, F. Creutzberg, F. R. Harris, A. G. McNamara, A.W. Yau, E. J. Llewellyn, D. Lummerzheim, M. H. Rees, I. C. McDade, and J. Margot, The ARIES auroral modelling campaign: characterization and modelling of an evening auroral arc observed from a rocket and a ground-based line of meridian scanners, Planet. Space Sci., 39, 1677-1705, 1991. 